\title{
INTERAÇÃO DE COMPOSTOS ORGANOSSULFURADOS DERIVADOS DO ALHO COM O CITOCROMO-C: UM ESTUDO ELETROQUÍMICO
}

Luisa del Carmen Barrett Reina, Carlos Alberto Montanari e Claudio Luis Donnici*

Departamento de Química/ICEx, Universidade Federal de Minas Gerais, Av. Antonio Carlos, 6627, 31270-901 Belo Horizonte - MG Izaura Cirino Nogueira Diógenes e Í́caro de Sousa Moreira

Departamento de Química Orgânica e Inorgânica, CC, Universidade Federal do Ceará, Campus do Pici, CP 12200, 60455-760

Fortaleza - CE

Recebido em 21/6/00; aceito em 27/6/01

\begin{abstract}
ON THE INTERACTION OF ORGANOSULFUR COMPOUNDS FROM GARLIC WITH CYTOCHROME-C: AN ELETROCHEMICAL STUDY. Organosulfur compounds present in garlic and onion have been evaluated as inhibitors of chemical carcinogenesis. Among them diallylsulfide was mainly investigated and studies demonstrated its metabolization to the corresponding sulfoxide and sulfone. In this work, we report the investigation of the interaction between the diallylsulfide and its oxidized derivatives, through cyclic voltammetry, with horse heart cytocrome-c (on a modified electrode with 4mercaptopyridine). Our results suggest that there is a reversible interaction between cytocrome-c and diallylsulfide and diallylsulfone and an irreversible interaction with the diallylsulfoxide.
\end{abstract}

Keywords: diallylsulfide derivatives; cyclic voltammetry; cytochrome-c.

\section{INTRODUÇÃO}

O câncer, segundo a Organização Mundial da Saúde, é a terceira causa mundial de óbitos (12\%), levando à morte cerca de 6,0 milhões de pessoas por ano no mundo, sendo 110 mil somente no Brasil. Estima-se que no nosso país cerca de 13 mil pessoas por ano devem morrer de câncer de estômago. Estudos epidemiológicos têm mostrado que populações com o hábito de consumo de grandes quantidades de alho e outros vegetais da família Allium apresentam baixa incidência de câncer, principalmente da região gástrica ${ }^{1-3}$. Além de sua utilidade gastronômica, tem-se estudado as aplicações do alho como antibiótico, anti-hipertensivo, anti-trombótico e na redução dos níveis de glicose no sangue 4 . Porém, só mais recentemente é que a sua eficiência como anti-cancerígeno foi descoberta e está sendo estudada ${ }^{2}$.

A inibição da carcinogênese por constituintes do alho tem sido observada em animais ${ }^{3}$. Acredita-se que a atividade anti-cancerígena do alho deve ocorrer principalmente pela presença de dialil-sulfeto (1) e de seus derivados oxidados dialil-sulfóxido (2) e dialil-sulfona (3). 1 é um tioéter lipofílico, obtido pela oxidação da alicina ${ }^{5}$, que demonstra eficiência na inibição de várias formas de câncer induzidas quimicamente; como exemplo pode-se citar a inibição da formação de tumores no cólon de ratos, provocados pela 1,2-dimetilidrazina ${ }^{6}$, e do câncer de esôfago em camundongos, induzido pelo $\mathrm{N}$-nitrosometilbenzilamina ${ }^{7}$. Postulase que a ação anti-cancerígena destes compostos tio-alílicos se deve à atuação sobre o sistema enzimático responsável pelo metabolismo de xenobióticos. Esta rota de metabolização pode ser resumida, no caso hipotético da metabolização de um alqueno simples $\left(\mathrm{RCH}=\mathrm{CH}_{2}\right)$, como a seguir: a) Fase I (Esquema 1) - ação oxidativa pela transferência de elétrons acompanhada por etapa química de oxidação (epoxidação ou hidroxilação) do xenobiótico $\left(\mathrm{RCH}=\mathrm{CH}_{2}\right)$ através da ação, por exemplo, de enzimas da família dos citocromos (CIT), como o citocromo P$450^{8}$ (cit-P450); ou o citocromo-c (cit-c) que pode agir metabolicamente, porém só é responsável pela transferência de elétrons ${ }^{9}$ aos substratos; b) Fase II (Esquema 1) - ação de famílias de enzimas (ex: glutationa-S-

* e-mail: cdonnici@dedalus.lcc.ufmg.br transferase, GST) encarregadas de adicionar um grupo hidrofílico (glutationa, GSH) facilitador da excreção ${ }^{10}$.

$$
\text { FASE I } \quad \mathrm{R}-\mathrm{CH}=\mathrm{CH}_{2}+\mathrm{O}_{2}+\mathrm{NADPH}+\mathrm{H}^{+} \stackrel{\mathrm{CIT}}{\longrightarrow} \stackrel{\mathrm{R}-\mathrm{CH}-\mathrm{CH}_{2}}{\stackrel{\mathrm{O}}{\mathrm{NADP}^{+}}+\mathrm{H}_{2} \mathrm{O}}
$$

Esquema 1. Possível mecanismo para o metabolismo de xenobióticos.

Há relatos que indicam que a atividade anti-cancerígena dos compostos tio-alílicos do alho está relacionada à inibição do cit-P450, o que impediria a oxidação do xenobiótico na Fase I, bloqueando a metabolização deste em seus metabólitos carcinogênicos ${ }^{6,7}$.

Outros estudos descritos na literatura mostram que há oxidação de 1 que é metabolizado a dialil-sulfóxido (2) e finalmente à dialilsulfona (3); posteriormente 3, na presença de NADPH, é metabolizada a outra espécie, um intermediário reativo ainda não identificado, que seria responsável pela inativação da enzima ${ }^{3 a}$. Além do mais, $\mathbf{1}, 2$ e 3 são inibidores competitivos do citocromo, sendo que $\mathbf{3}$ age sobre a enzima mais rapidamente.

Assim, os organossulfurados devem agir sobre o citocromo ou em uma primeira etapa única de transferência de elétrons (etapa I.a, Esquema 2) - como com a ação de cit-c - ou em uma etapa de transferência de elétrons seguida de oxidação química (etapas Ia e I.b, Esquema 2)- como com a ação de cit-P450.

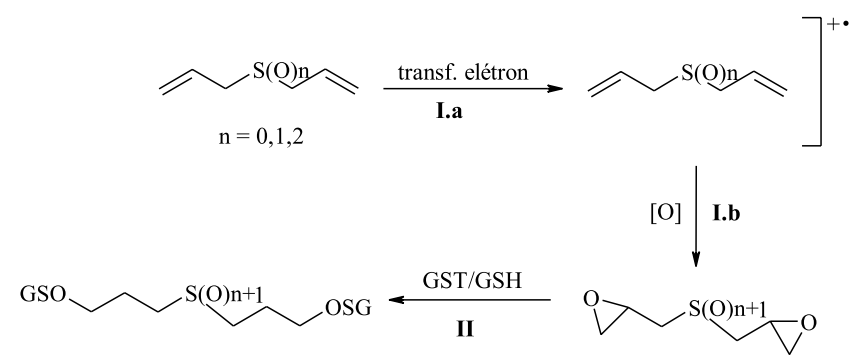

Esquema 2. Possíveis rotas de metabolismo de organossulfurados alílicos. 
Desde que a atividade anti-cancerígena dos compostos tio-alílicos presentes no alho ainda não foi adequadamente esclarecida, o objetivo deste trabalho é o estudo do comportamento eletroquímico de dialil-sulfeto (1) e dos correspondentes sulfóxido 2 e sulfona 3 na presença do citocromo-c (cit-c), responsável somente pelo transporte de elétrons no metabolismo. Pelo acompanhamento (através da técnica de voltametria cíclica) do processo reversível de transferência de elétrons do cit-c, pode-se observar a alteração causada pela adição de cada organossulfurado no processo redox do cit-c e estas informações eletroquímicas mostrariam a existência, ou não, de interação destes compostos com o cit-c, além de sugerir qual a influência da primeira etapa de transferência de elétrons no metabolismo dos compostos tio-alílicos do alho. Indiretamente, destes dados poder-se-ia também sugerir se há correlação entre o mecanismo de metabolização e o mecanismo de ação anti-cancerígena destes compostos.

\section{PARTE EXPERIMENTAL}

Os espectros de $\mathrm{RMN}$ de ${ }^{1} \mathrm{H}$ e de ${ }^{13} \mathrm{C}$ foram obtidos no espectrofotômetro Bruker $200 \mathrm{MHz}$ no Departamento de Química da UFMG. Os espectros na região do infravermelho (IV) foram obtidos em um interferométrico Mattson Instruments Galaxy 3000 na faixa de $400 \mathrm{a} 4000 \mathrm{~cm}^{-1}$. Os espectros em filme líquido foram obtidos utilizando-se janelas de $\mathrm{NaCl}$ ou celas de $\mathrm{NaCl}$ de $0,025 \mathrm{~mm}$ de espessura, e para sólidos, foram obtidos na forma de pastilhas de $\mathrm{KBr}$. As análises cromatográficas foram efetuadas em um equipamento CG Shimadzu, modelo 17-A, equipado com um integrador digital eletrônico, utilizando um detetor de ionização de chama. $\mathrm{O}$ gás de arraste empregado foi nitrogênio, e a coluna: fase líquida DB1 dimetilpolisilixano com $30 \mathrm{~m}$ de comprimento e $0,25 \mathrm{~mm}$ de diâmetro interno. As condições usuais de programação de análise foram temperatura inicial de $50^{\circ} \mathrm{C}$ por 5 minutos, e aumento de $10{ }^{\circ} \mathrm{C}$ por minuto até $250^{\circ} \mathrm{C}$. Os pontos de fusão foram determinados em aparelho Fisher-Johns, sem correção. Os espectros eletrônicos na região do visível e do ultravioleta foram obtidos em um espectrofotômetro Hitachi, modelo U-2000 de duplo feixe. O acompanhamento espectroscópico foi feito através da observação da banda em 695 nm atribuída ${ }^{11}$ à transição LMCT (Ligand to Metal Charge Transfer) dos orbitais $\mathrm{p} \pi$ do átomo de enxofre do resíduo metionina 80 para os orbitais $\mathrm{d} \pi$ do átomo de Fe do grupo heme do cit-c.

\section{Reagentes}

O dialil-sulfeto 1 (Merck) e os compostos derivados sulfóxido 2 e sulfona $\mathbf{3}$, com suas preparações descritas a seguir, tiveram a sua caracterização efetuada por cromatografía gasosa e métodos espectrométricos na região do infravermelho e de $\mathrm{RMN}$ de ${ }^{1} \mathrm{H}$ e de ${ }^{13} \mathrm{C}$.

Preparação do dialil-sulfóxido $(2)^{12}$

Conforme descrito, a partir de 2,2 $\mathrm{mL}$ de $\mathbf{1}$ (1,96 g; 17,0 mmoles) e solução $0,5 \mathrm{~mol} \mathrm{~L}^{-1}$ de $\mathrm{NaIO}_{4}(4,3 \mathrm{~g} ; 20$ mmoles) em acetonitrila obteve-se 2,13 g (16,3 mmoles; $96 \%$ ) de um óleo claro.

IV (filme, $\left.\mathrm{cm}^{-1}\right)$ : $1639\left(v_{\mathrm{C}=\mathrm{C}}\right) ; 993,939\left(v \mathrm{CH}_{2}=\mathrm{CH}-\mathrm{CH}_{2}\right) ; 1050$ $\left(\mathrm{n}_{\mathrm{S}=\mathrm{O}}\right) ; \mathrm{RMN}$ de ${ }^{1} \mathrm{H}(200 \mathrm{MHz})-\delta\left(\mathrm{CDCl}_{3} / \mathrm{TMS}\right): 5,97-5,79(\mathrm{~m}, 2 \mathrm{H})$; $5,49-5,34(\mathrm{~m}, 4 \mathrm{H}) ; 3,59-3,25(\mathrm{~m}, 4 \mathrm{H})$; RMN de ${ }^{13} \mathrm{C}(200 \mathrm{MHz})-\delta$ ( $\left.\mathrm{CDCl}_{3} / \mathrm{TMS}\right): 125,60 ; 123,55 ; 54,10$

\section{Preparação da dialil-sulfona $(\mathbf{3})^{13}$}

Partindo-se de 1 (8,01g; 70,3 mmoles) e reagindo-se com $21,6 \mathrm{~mL}$ de $\mathrm{H}_{2} \mathrm{O}_{2}$ à $30 \%$ em $180 \mathrm{~mL}$ de ácido acético glacial, con- forme descrito, obteve-se um óleo $(8,37 \mathrm{~g}, 82 \%)$ após destilação a pressão reduzida (176-178 ${ }^{\circ} \mathrm{C} / 50$ Torr).

IV (filme, $\left.\mathrm{cm}^{-1}\right): 1639\left(v_{\mathrm{C}-\mathrm{C}}\right) ; 993,939\left(v \mathrm{CH}_{2}=\mathrm{CH}-\mathrm{CH}_{2}\right) ; 1325$, $1137\left(\mathrm{nSO}_{2}\right) ; \mathrm{RMN}$ de ${ }^{1} \mathrm{H}(200 \mathrm{MHz})-\delta$ ( $\left.\mathrm{CDCl}_{3} / \mathrm{TMS}\right): 6,03-5,83$ $(\mathrm{m}, 2 \mathrm{H}) ; 5,54-5,39(\mathrm{~m}, 4 \mathrm{H}) ; 3,71(\mathrm{~d}, 4 \mathrm{H}, \mathrm{J}=7 \mathrm{~Hz}) \mathrm{RMN} \mathrm{de}{ }^{13} \mathrm{C}$ (200MHz) - $\delta\left(\mathrm{CDCl}_{3} / \mathrm{TMS}\right): 124,77 ; 124,74 ; 55,89$

A metaloproteína citocromo c de coração de cavalo (cit-c) utilizada foi a do tipo VI (Aldrich- purificada por método Brautigan ${ }^{14}$ ); solução eletrolítica: tampão fostato $\left(\mathrm{KH}_{2} \mathrm{PO}_{4}\right) 100 \mathrm{mM}, \mathrm{pH}=7,0$; promotor: 4-mercaptopidirina, (Aldrich).

\section{Eletroquímica}

Medidas eletroquímicas: analisador eletroquímico Bioanalytical Systems modelo BAS 100BW interfaciado a um computador PC 486; celas eletroquímicas de vidro convencional de três eletrodos tendo como trabalho ouro policristalino (BAS, $A=0,0314 \mathrm{~cm}^{2}$ ) modificado com o ligante 4-mercaptopiridina (pyS), placa de Pt e prata/cloreto de prata ( $\mathrm{KCl} 3,5 \mathrm{~mol} \mathrm{~L}^{-1}$-BAS) como auxiliar e referência, respectivamente. $\mathrm{O}$ eletrodo de trabalho foi preparado através da imersão, por 15 minutos, do eletrodo de ouro policristalino em uma solução aquosa saturada do ligante pyS. Após esse período, a superfície foi lavada exautivamente com água desionizada, e imediatamente empregada nos experimentos eletroquímicos. Eletrodos modificados com pyS, obtidos por este procedimento, são largamente empregados nos estudos eletroquímicos de metaloproteínas ${ }^{15-17}$, razão pela qual podem ser considerados convencionais para tais fins. Os eletrodos são considerados estáveis na escala de tempo dos experimentos eletroquímicos realizados ${ }^{16}$, e são normalmente caracterizados por métodos eletroquímicos ${ }^{18,19}$ e por espectroscopia vibracional SERS (Surface Enhanced Raman Scattering) ${ }^{20,21}$ e na região do infravermelho ${ }^{22}$.

Para evitar contaminação, celas eletroquímicas distintas foram utilizadas com a finalidade de se estudar o efeito da adição dos diferentes compostos organossulfurados no processo redox do cit-c. As soluções de trabalho, contendo cit-c, foram estocadas em refrigerador à $4{ }^{\circ} \mathrm{C}$. Os ciclo-voltamogramas foram obtidos a velocidade de varredura de $50 \mathrm{mV} \mathrm{s}^{-1}$ na região de potencial de $-0,4 \mathrm{~V}$ a $+0,4 \mathrm{~V}$ vs $\mathrm{Ag} / \mathrm{AgCl}$ ( $\mathrm{KCl} 3,5 \mathrm{~mol} \mathrm{~L}^{-1}$-BAS), sentido anódico; início da varredura em $-0,4 \mathrm{~V} .+(\mathrm{i}=0, \mathrm{E}=0)$. Os experimentos eletroquímicos realizados com a metaloproteína cit-c seguiram o seguinte procedimento: (i) limpeza do eletrodo de Au policristalino de área geométrica $0,0314 \mathrm{~cm}^{2}$ em solução "piranha" $\left(3 \mathrm{H}_{2} \mathrm{SO}_{4}: 1 \mathrm{H}_{2} \mathrm{O}_{2}\right)$ (CUIDADO: SOLUÇÃO EXTREMAMENTE OXIDANTE, EVITAR CONTATO COM A PELE); (ii) lavagem com $\mathrm{H}_{2} \mathrm{O}$ desionizada; (iii) polimento em pasta de alumina de diferentes granulações até obtencão de uma superfície especular; (iv) submetido a ultra-som por $15 \mathrm{mi}$ nutos em $\mathrm{H}_{2} \mathrm{O}$ desionizada; (v) imersão de quinze minutos em solução saturada de 4-mercaptopiridina, pyS; (vi) voltametria cíclica em solução $1 \mathrm{mmol} \mathrm{L}^{-1}$ de cit-c em $\mathrm{KH}_{2} \mathrm{PO}_{4} 100 \mathrm{mmol} \mathrm{L}^{-1}, \mathrm{pH}=7,0$, volume da cela $=1 \mathrm{~mL}$.

\section{RESULTADOS E DISCUSSÃO}

\section{Resultados eletroquímicos com dialil-sulfeto (1)}

Os ciclo-voltamogramas da solução de cit-c, com e sem a adição de 1, encontram-se ilustrados na Figura 1. Os valores de $\mathrm{E}_{1 / 2}$ e de $\Delta \mathrm{E}_{\mathrm{p}}$, após adição de $\mathbf{1}$ (Figura 1, curvas $\mathbf{b}$ e c), permanecem próximos àqueles observados para a proteína in natura ${ }^{23}$ (Figura 1, curva a), independente da quantidade e do tempo de adição deste composto tioalílico; mesmo após 48 horas não se observou qualquer alteração. 


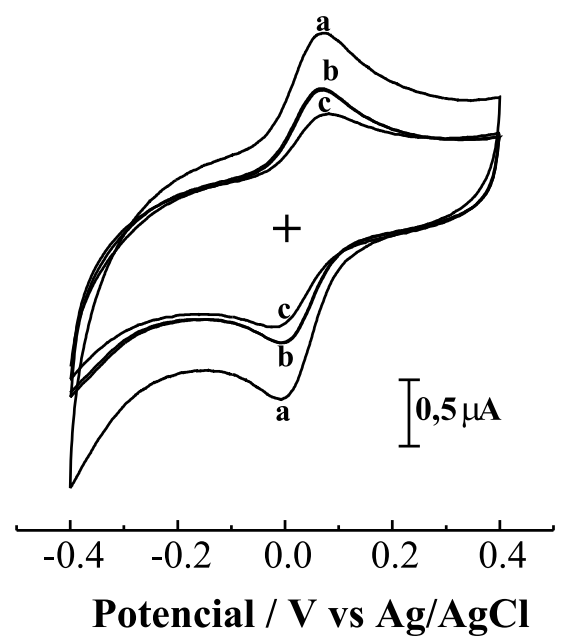

Figura 1. Ciclo-voltamogramas a $50 \mathrm{mV} \mathrm{s}^{-1}$ em $\mathrm{KH}_{2} \mathrm{PO}_{4} 100 \mathrm{mmol} \mathrm{L}^{-1}, \mathrm{pH}=$ 7,0 de (a) cit-c $1 \mathrm{mmol} \mathrm{L} \mathrm{L}^{-1}$ e deste após 48h da adição de $\mathbf{I}$ (b) $77 \mu \mathrm{mol} \mathrm{L} \mathrm{L}^{-1} \quad$ e (c) $385 \mu \mathrm{mol} \mathrm{L} L^{-1}$. Eletrodo de trabalho: Au modificado com o ligante pyS.

De acordo com a equação de Cottrell $^{24}$ para espécies em difusão em solução esperar-se-ia uma diminuição menos pronunciada do valor de $i_{p c}$ em função da diminuição da concentração de cit-c decorrente da adição de 1. A diminuição observada nos valores de $i_{p c}$ pode ser atribuída ao processo de adsorção competitiva do sulfeto $\mathbf{1}$, o que diminuiria a eficiência do modificador pyS em acessar a reação heterogênea de transferência de elétrons do cit-c. Esta atribuição foi feita com base na manutenção dos valores absorciométricos da banda LMCT observada em $695 \mathrm{~nm}$.

Resultados registrados na literatura ${ }^{25,26}$ sobre a eletroquímica do cit-c, indicaram que para que a interação entre o promotor e o cit-c seja efetiva é necessário: (i) que o promotor possua um sítio de interação com a superfície (grupo funcional X); (ii) uma superfície hidrofílica com sítios de interação básicos ou, no mínimo, não repulsivos (grupo funcional Y) e (iii) uma superfície que não permita a adsorção degradativa do cit-c.

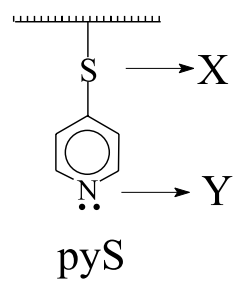

Portanto, a adsorção de $\mathbf{1}$ ao eletrodo de ouro, através do átomo de enxofre, poderia levar à modificação da superfície tornando-a inadequada para a análise eletroquímica do cit-c. Neste caso ter-se-ia, provavelmente, a formação de uma monocamada irregular constituída por pyS e 1 o que, consequentemente, diminuiria os sítios de interação da superfície com o cit-c e, portanto, também diminuiria a corrente do processo redox em questão.

A observação de um processo reversível, mesmo após a adição do composto 1 à solução de cit-c, poderia sugerir que não há interação entre estes, por não terem sido evidenciadas alterações eletroquímicas desta metaloproteína. Entretanto, a literatura registra que tal interação com o citocromo só é possível quando há uma etapa química consecutiva à transferência de elétrons, como com o uso de citocromo $\mathrm{P}$ 450; quando esta etapa não é observada, não há prosseguimento do metabolismo do xenobiótico ${ }^{8,9}$.

Vale ressaltar, ainda, que a interação por processo de transferência de elétrons reversível entre ligantes sulfurados (tióis e sulfetos) e o grupo ferro-heme de porfirinas ${ }^{27}$ é conhecida e não necessariamente conduz a alterações espectroscópicas, especialmente com sulfetos, como o caso de 1. Assim, pode-se sugerir a ocorrência de transferência reversível de elétrons entre 1 e o cit-c (etapa I.a, Esquema 2) sem a etapa oxidativa química posterior (etapa I.b, Esquema 2).

\section{Resultados eletroquímicos com dialil-sulfóxido (2)}

Os ciclo-voltamogramas da solução de cit-c com e sem 2 encontram-se ilustrados na Figura 2. Os resultados obtidos indicam, claramente, uma acentuada diferença após 1 hora da adição de $\mathbf{2}$, observando-se o desaparecimento do processo faradáico do cit-c após $7 \mathrm{~h}$ da adição.

Após obtenção da curva (c), ilustrada na Figura 2, retirou-se o eletrodo de trabalho da solução, lavou-se exaustivamente com água desionizada e etanol e, em seguida, efeturaram-se varreduras de potencial em uma solução recém-preparada de cit-c em $\mathrm{KH}_{2} \mathrm{PO}_{4}$ $100 \mathrm{mmol} \mathrm{L}^{-1}$. O ciclo-voltamograma obtido foi semelhante à curva (a) da Figura 2. Dessa forma fica descartada a possibilidade de adsorção competitiva do composto 2 que tornaria inadequada a análise eletroquímica do cit-c.

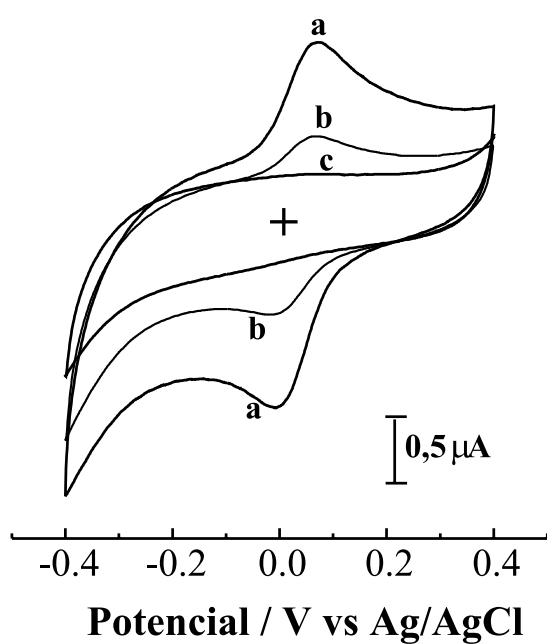

Figura 2. Ciclo-voltamogramas a $50 \mathrm{mV} \mathrm{s}^{-1}$ em $\mathrm{KH}_{2} \mathrm{PO}_{4} 100 \mathrm{mmol} \mathrm{L}^{-1}$, pH = 7,0 de (a) cit-c 1 mmol $L^{-1}$ e deste após (b) 1 h da adição de 2, 68 rmol $L^{-1} e$ (c) $7 \mathrm{~h}$ da adição de 2, $68 \mu \mathrm{mol} \mathrm{L}^{-1}$. Eletrodo de trabalho: Au modificado com o ligante pyS.

As variações espectroscópicas da banda LMCT em $695 \mathrm{~nm}$ do cit-c com o tempo de adição de $\mathbf{2}$ encontram-se ilustrados na Figura 3. A diminuição progressiva nos valores de absorvância até seu desaparecimento, após $24 \mathrm{~h}$ de adição do composto 2 , indicam que há desnaturação da metaloproteína. A banda Soret, em $410 \mathrm{~nm}$, entretanto, não experimenta variações significativas. Isto tem sido observado, também, quando do processo de digestão do cit-c pela tripsina ${ }^{28}$. Cabe ressaltar que este tipo de interação cit-c/sulfóxido deve conduzir à coordenação axial irreversível entre o átomo de oxigênio muito básico do grupo sulfóxido e o grupo ferro-heme de citocromo levando de fato à perda de atividade, conhece-se por exemplo o ataque nucleofílico de espécie oxigenada sobre porfirinas sintéticas ${ }^{29}$.

\section{Resultados eletroquímicos com dialil-sulfona (}

Os ciclo-voltamogramas da solução de cit-c com e sem 3 encontram-se ilustrados na Figura 4. Os valores de $\mathrm{E}_{1 / 2}$ e de $\Delta \mathrm{E}_{\mathrm{p}}$, após adição de 3 (Figura 4 , curva c), permanecem próximos àqueles observados 


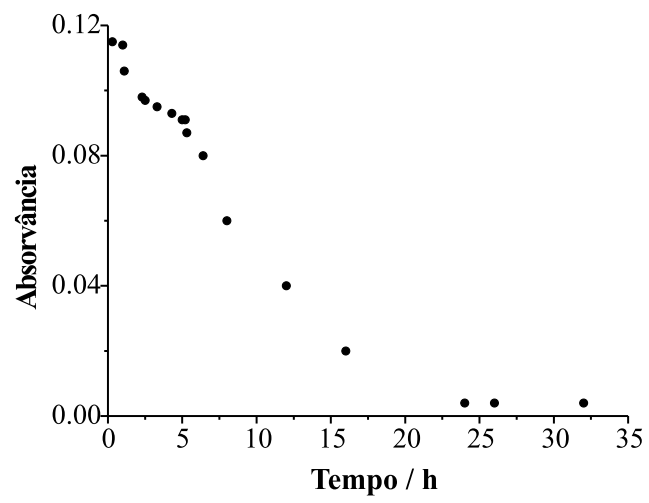

Figura 3. Gráfico de absorvância vs tempo da solução de cit-c após adição de 2, $544 \mu$ mol $L^{-1}$.

para a proteína in natura ${ }^{23}$ (figuras 1 e 4), independente da quantidade e do tempo de adição de $\mathbf{3}$ e como no caso de $\mathbf{1}$, mesmo após 48 h não há alteração alguma. Além do mais, os resultados espectroscópicos obtidos não apresentaram variação no valor de absorvância da banda em $695 \mathrm{~nm}$, mesmo após $48 \mathrm{~h}$ de reação, o que indica que a proteína não experimenta desnaturação após adição de 3, tal como observado para 1 . Como no caso do sulfeto 1 , pode-se sugerir que há uma interação por transporte de elétrons reversível entre o sulfóxido 3 e o cit-c.

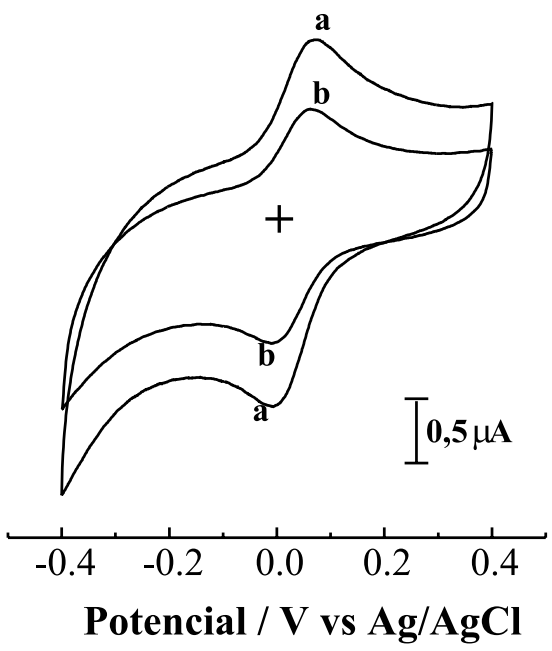

Figura 4. Ciclo-voltamogramas a $50 \mathrm{mV} \mathrm{s}^{-1} \mathrm{em} \mathrm{KH}_{2} \mathrm{PO}_{4} 100 \mathrm{mmol} \mathrm{L}^{-1}$, $p H=7,0$ de (a) cit-c $1 \mathrm{mmol} L^{-1}$ e deste após (b) $48 \mathrm{~h}$ da adição de $\mathbf{3}$, 61 umol $L^{-1}$. Eletrodo de trabalho: Au modificado com o ligante pyS.

\section{Experiências com ampliação da faixa de potencial de varredura}

Armstrong e colaboradores ${ }^{30}$ observaram que formas desnaturadas da metaloproteína cit-c apresentavam processos redox com valores de potencial formal de meia onda, $\mathrm{E}_{1 / 2}$, próximos a $-500 \mathrm{mV}$ vs $\mathrm{Ag}$ / $\mathrm{AgCl}$. Os ciclo-voltamogramas ilustrados na Figura 5 foram obtidos com o objetivo de verificar o efeito da adição de $\mathbf{1}$ ou $\mathbf{3}$ na estrutura física desta metaloproteína.

Iniciando-se as curvas voltamétricas no limite catódico da varredura, - 0,4 e - $-0,7 \mathrm{~V}$ nas curvas (a) e (b), respectivamente, é possível observar a onda de oxidação do par redox $\mathrm{Fe}^{\mathrm{III} / \mathrm{II}}$ do grupo heme do citc, próximo a 0,0 V. A onda de redução, entretanto, não é mais observada nas curvas em que o limite anódico ultrapassa o valor de $+1,0 \mathrm{~V}$, ou seja, o início do processo de oxidação da superfície de $\mathrm{Au}$, conforme ilustrado na Figura 5. Neste caso, tem-se o processo de dessorção física da monocamada do modificador pyS. As diferenças observadas entre as curvas (b) e (c) da Figura 5 são atribuídas ${ }^{21}$ à modificação da superfície de ouro com o ligante organossulfurado pyS; o pico a $+0,5 \mathrm{~V}$ na curva (b) é referente à redução do modificador py $S^{21,31}$.

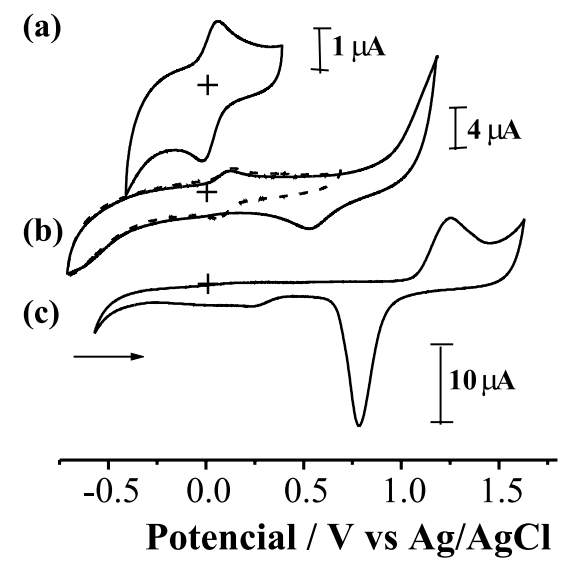

Figura 5. Ciclo-voltamogramas a $50 \mathrm{mVs}^{-1}$ em $\mathrm{KH}_{2} \mathrm{PO}_{4} 100 \mathrm{mmol} \mathrm{L} \mathrm{m}^{-1}$, $p H=7,0$ de (a) cit-c $1 \mathrm{mmol} L^{-1} e(b)$ cit-c 1 mmol $L^{-1}$, na presença de $385 \mu \mathrm{mol} \mathrm{L} L^{-1}$ de 1. Eletrodo de trabalho: Au modificado com o ligante pyS. (c) Ciclo voltamograma a $50 \mathrm{mVs}^{-1}$ em $\mathrm{KH}_{2} \mathrm{PO}_{4} 100 \mathrm{mmol} \mathrm{L}^{-1}, \mathrm{pH}=7,0$. Eletrodo de trabalho: Au limpo.

A limitação da faixa de potencial de trabalho é imposta pelo modificador (pyS), uma vez que a monocamada deste é destruída logo após o início da formação do óxido de ouro ( 1,0 V, Figura 5). $\mathrm{O}$ pico observado em $+0,8 \mathrm{~V}$ (Figura 5 , curva c) é atribuído ${ }^{31}$ à redução do óxido de ouro. Resultados ${ }^{20}$ de espectroscopia SERS (Surface Enhanced Raman Scattering), da superfície de ouro modificada com o ligante pyS reforçam a atribuição de destruição física da monocamada nos limites da varredura anódica e catódica, $+0,9$ e $0,6 \mathrm{~V}$, respectivamente.

Os resultados ilustrados na curva (b) da Figura 5, portanto, reforçam a atribuição da ausência do processo de desnaturação da metaloproteína cit-c após a adição do sulfeto $\mathbf{1}$ e da sulfona $\mathbf{3}$, uma vez que não foi observada a onda voltamétrica próxima a $-500 \mathrm{mV}$ e portanto, corrobora-se a hipótese da ocorrência da transferência reversível de elétrons entre o cit-c e $\mathbf{1}$ e $\mathbf{3}$.

Experimentos para melhor investigação do comportamento eletroquímico foram efetuados variando-se a velocidade de varredura. A verificação da presença de efeitos cinéticos depende do tempo em que o experimento é realizado ${ }^{24}$. Em baixas velocidades de varredura (longos períodos), os sistemas, normalmente, apresentam um comportamento reversível, ao passo que em elevadas velocidades (períodos curtos) se observa, em geral, um comportamento irreversível. Quando se utiliza a técnica de voltametria cíclica ${ }^{15}$ o par redox que apresenta uma cinética heterogênea de transferência de elétrons só permite, em geral, a aquisição de ciclo-voltamogramas interpretáveis até o limite de velocidade de varredura de $500 \mathrm{mVs}^{-1}$.

De fato, conforme resumido na Tabela 1 , com e sem a adição dos compostos organossulfurados $\mathbf{1}$ e $\mathbf{3}$ as características descritas acima são observadas durante o estudo voltamétrico do cit-c. Além do mais, os resultados descritos na Tabela 1 para as curvas obtidas para o cit-c nas velocidades citadas, sugerem a ausência de mecanismo EC (etapa eletroquímica seguida de etapa química) e a existência de um processo difusional com características de reversibilidade ${ }^{24}$, apesar da queda ôhmica evidenciada. 
Tabela 1. Valores de $\Delta \mathrm{E}_{\mathrm{p}}(\mathrm{mV})$ do processo redox do cit-c em diferentes velocidades de varredura.

\begin{tabular}{cc}
\hline $\mathbf{v}\left(\mathbf{V s}^{-\mathbf{1}}\right)$ & $\Delta \mathbf{E}_{\mathbf{p}}(\mathbf{m V})$ \\
\hline 0,05 & 75 \\
0,1 & 93 \\
0,2 & 84,7 \\
0,3 & 99,1 \\
0,4 & 100,9 \\
\hline
\end{tabular}

No caso do sulfóxido 2 não foi possível fazer esse estudo já que, logo após a adição do mesmo à solução de cit-c, não mais foi possível observar o processo redox deste.

$\mathrm{Na}$ tentativa de se observar algum outro processo químico acoplado, nos casos de $\mathbf{1}$, e de $\mathbf{3}$, de cinética mais lenta, fizeram-se varreduras de potencial de 1 a $20 \mathrm{mVs}^{-1}$. $\mathrm{O}$ único processo redox observado, entretanto, foi o do cit-c, próximo de $0,0 \mathrm{~V}$.

\section{Racionalização Mecanística}

Os resultados voltamétricos e espectroscópicos obtidos indicam que:

(i) Apesar de se observar comportamento eletroquímico similar para 1 e 3, o dialil-sulfeto $\mathbf{1}$, por possuir elétrons não-ligantes no átomo de enxofre para quelação, pode sofrer um processo de adsorção, diminuindo-se a eficiência do modificador pyS em acessar a reação heterogênea de transferência de elétrons do cit-c. A dialil-sulfona 3 , não possui elétrons nãoligantes no átomo de enxofre para a quelação e não deve sofrer adsorção em superfície de ouro nem interage com a metaloproteína em solução.

(ii) Os experimentos realizados em velocidades de varredura de $1 \mathrm{a} 400 \mathrm{mVs}^{-1}$ sugerem que não há reação química acoplada ao processo redox do cit-c na faixa de potencial de $-0,7$ a $+0,7 \mathrm{~V}$ vs $\mathrm{Ag} / \mathrm{AgCl}$.

(iii) As hipóteses (i) e (ii) não excluem a ocorrência de um processo de transferência de elétrons reversível entre o sulfeto 1 e a sulfona 3 com o cit-c.

(iv) A ausência do processo redox, assim como o desaparecimento da banda observada em $695 \mathrm{~nm}$ no espectro eletrônico, após adição do dialil-sulfóxido (2) à solução de cit-c, indica que há interação entre 2 e o cit-c. É provável que este processo ocorra através da coordenação do átomo de enxofre do grupo sulfóxido ao Fe do grupo heme, o qual experimenta uma reação de substituição do resíduo metionina 80 do cit-c e/ou de ambos os sítios axiais: histidina $18 \mathrm{e}$ metionina $80^{30}$.

\section{CONCLUSÕES}

Finalmente, pelos resultados voltamétricos obtidos, pode-se concluir que o comportamento eletroquímico do cit-c, com processo reversível de transferência de elétrons, só é alterado na presença do sulfóxido 2 através de uma interação irreversível com o cit-c. Desde que dados da literatura mostram que 1, 2 e 3 são inibidores competitivos do citocromo, com a sulfona $\mathbf{3}$ sendo a mais reativa, os nossos dados sugerem que não há influência do processo de transferência de elétrons (etapa I.a, Esquema 2) no metabolismo dos compostos organossulfurados do alho pois, se assim fosse ter-se-ia observado um comportamento similar entre os três derivados e $\mathbf{3}$ apresentaria a melhor interação. Pode-se sugerir também que não deve haver correlação entre a primeira etapa de transferência de elétrons da metabolização e o mecanismo de ação anti-cancerígena destes compostos tioalílicos do alho.

\section{AGRADECIMENTOS}

Os autores agradecem à CAPES, CNPq, FAPEMIG, FINEP, FUNCAP e PADCT pelo apoio financeiro e pela bolsa concedida (CNPq).

\section{REFERÊNCIAS}

1. Block, E.; Angew. Chem. Int. Ed. Engl. 1990, 31, 1135.

2. Wargovich, M. J.; Uda, N.; Word, C.;Velasco, M.; Mckee, K.; Biochem. Soc. Trans. 1996, 24, 811 .

3. (a)Yang, C. S.; Wang, Hi-Y; Harg, J.Y.; Diet and Cancer: Markers, Prevention and Treatment 1994, 113; (b) Brady, J. F.; Wang, M. H.; Hong, J.Y.; Xiao, F.; Li, Y.; Yoo, J.S.H.; Ning, S. M.; Lee, M. J.; Fukuto, J.M., Gapac, J. M.; Yang, C.S.; Toxicol. Appl. Pharm. 1991, 108, 342; (c) Gudy, V. A.; Singh, S.V.; Biochem. Pharm. 1991, 42, 1261; (d) Wattenberg, L. W.; Sparnins, V. L.; Barany, G.; Cancer Res. 1989, 49, 2689.

4. O'Brien, J.; Trends Food Sci. Tech. 1990, 155.

5. Wargovich, M. J.; Imada, E; Stephens, L. C.; Cancer Lett. 1992, 64, 39.

6. Wargovich, M. J.; Carcinogenesis 1987, 8, 487

7. Wargovich, M. J.; Woods, C.; Eng, V. W. S.; Stephens, L. E.; Gray, K.; Cancer Res. 1988, 48, 6872.

8. Quici, S.; Banfi, S.; Pozzi, G.; Gazz. Chim. Ital. 1993, 123, 597.

9. Wijesekara, T. P.; Dolphin, D.; In Metalloporphyrins in Catalytic Oxidations (R. A. Sheldon,ed.), Marcel Dekker, New York 1994.

10. Dirr, H.; Reinemer, P. E.; Huber, R.; Eur. J. Biochem. 1994, $220,645$.

11. Kuznetsov, B. A.; Shumakovich, G. P.; Mestechkina, N. M.; Bioelectrochem. Bioenerg. 1977, 4, 512.

12. Russel, G. A.; Ochrymowycz, L. A.; J. Org. Chem. 1970, 35, 2106.

13. Backer, H. J.; Stating, J.; Hazenberg, J. F. A.; Tetrahedron 1986, 42, 54.

14. Brautigan, D. L.; Fergusson-Miller, S.; Margoliash, E. In Methods in Enzimology Vol. LIII, Fleischer, S.; Pucker, L. Eds., Academic Press: New York, 1978.

15. Hawkridge, F. M.; Taniguchi, I.; Comments on Inorg. Chem. 1995, 17, 163.

16. Allen, P. M.; Hill, H. A. O.; Walton, N. J.; J. Electroanal. Chem. 1984, $178,69$.

17. Diógenes, I. C. N.; Nart, F. C.; Moreira, I. S.; Inorg. Chem. 1999, 108, 3335 .

18. Hinnen, C.; Niki, K.; J. Electroanal. Chem. 1989, 264, 157.

19. Zhong, C.-J.; Porter, M. D.; J. Am. Chem. Soc. 1994, 116, 11616.

20. Jun, F. K-.; Satake, I.; Ueda, K.; Akutsu, H.; Niki, K.; Redox Chemistry and Interfacial Behaviour of Biological Molecules, 1988, 125.

21. Taniguchi, I.; Iseki, M.; Toyosawa, K.; Yamaguchi, H.; Yasukouchi, K.; J. Electroanal. Chem. 1985, 186, 299.

22. Lamp, B. D.; Hobara, D.; Porter, M. D.; Niki, K.; Cotton, T. M.; Langmuir, 1997, 13, 736.

23. Eddowes, M. J.; Hill, H. A.; J. Chem. Commun. 1977, 771.

24. Bard, A. J.; Faulkner, L. R.; Electrochemical Methods, Fundamentals and Applications; John Wiley \& Sons: New York, NY, 1980.

25. Allen, P. M.; Hill, H. A. O.; Walton, N. J.; J. Electroanal. Chem. 1984, 69, 1778.

26. Sellers, H.; Ulman, A.; Shnidman, Y.; Eilers, J. E.; J. Am. Chem. Soc. 1993, 115,9389

27. (a) Dawson, J.H.; Sono, M.; Chem. Rev. 1987, 87, 1255; (b) Miller, V.P.; DePillis, G.D.; Ferrer, J.C.; Mauk, A.G.; Montellano, P.R.O.; J. Biol. Chem. 1992, 267, 8936 .

28. Moreira, I. S.; Sun, J.; Cho, M. O. K.; Wishart, J. F.; Isied, S. S.; J. Am. Chem. Soc. 1994, 116, 8396.

29. Cohen, I. A., Chow, B. C.; Inorg. Chem. 1974, 13, 488.

30. Armstrong, F. A; Struct. Bonding 1990, 72, 137.

31. Taniguchi, I.; Iseki, M.; Toyosawa, K.; Yamaguchi, H.; Yasukouchi, K.; J. Electroanal. Chem. 1984, 164, 385. 